\title{
GLOCALIZACIÓN Y USO DEL ESPACIO EN LAS ADAPTACIONES CINEMATOGRÁFICAS SURCOREANAS DE OBRAS LITERARIAS EXTRANJERAS. LOS CASOS DE BURNING Y LA DONCELLA
}

\section{Glocalization and Use of Space In South Korean Film Adaptations of Foreign Literary Works. The cases of Burning and The Handmaiden}

\author{
Álvaro TRIgO MALDONADO \\ Universidad de Salamanca \\ alvarotrigo@usal.es
}

Recibido: 15/03/2021; Aceptado: 21/04/2021; Publicado: 31/12/2021

Ref. Bibl. ÁLVARO TRIGO MALDONADO. GLOCALIZACIÓN Y USO DEL ESPACIO EN LAS ADAPTACIONES CINEMATOGRÁFICAS SURCOREANAS DE OBRAS LITERARIAS EXTRANJERAS. LOS CASOS DE BURNING Y LA DONCELLA. 1616: Anuario de Literatura Comparada, 11 (2021), 203-218

RESUMEN: El presente artículo analiza las estrategias narrativas utilizadas en dos adaptaciones surcoreanas al cine de obras literarias extranjeras. Las obras seleccionadas han sido los thrillers psicológicos Burning (Lee Changdong, 2018) y La doncella (Park Chan-wook, 2016) basados en obras literarias de Haruki Murakami y Sarah Waters respectivamente. A lo largo del ensayo se pone especial énfasis en la utilización de los espacios y los cambios narrativos para contextualizar la forma en que estos dos nuevos productos resultantes continúan articulándose en torno a un enfoque glocalizador que apela a la identidad colec- 
tiva de los coreanos dando así continuidad a una estrategia de consumer nationalism muy ligada tanto al desarrollo de la industria cinematográfica surcoreana como al de su nacionalismo. También se argumentará la manera en que las dos películas que surgen de lo que podríamos denominar traducción cultural no se limitan a una imitación de los materiales de origen, sino que añaden diversas dimensiones y profundidad nuevas convirtiéndose en obras de pleno derecho.

Palabras clave: cine coreano; estudios de adaptación; cine y literatura; Burning; La doncella.

ABSTRACT: This paper analyzes how the narratives of two Korean contemporary cinema productions based on foreign literary works have given birth to products that continue to articulate themselves around the local and the appeal to a collective national identity following a strategy of glocalization adopted by the South Korean cinema industry. Two recent psychological thrillers that share this characteristic have been selected: Lee Changdong's Burning (2018) and Park Chan-wook's The Handmaiden (2016) based on the works of Haruki Murakami and Sarah Waters respectively. Through this essay it is emphasized how both adaptations use the spaces and narrative changes to pursue a glocalization approach that appeals to a shared Korean identity continuing a strategy of "consumer nationalism" which has been intrinsically related to both the film industry in South Korea and the historical development of Korean nationalism. It will be also argued how the two new products that emerge from this cultural translation do not limit themselves to an imitation of the source materials and go beyond them adding new depth and dimensions thus constituting full new works.

Key words: Korean cinema; adaptation studies; literature and film; Burning; The Handmaiden.

\section{INTRODUCCIÓN}

A lo largo del siguiente trabajo se analizarán dos adaptaciones recientes de obras literarias extranjeras en Corea del Sur y se reflexionará de qué manera apelan a un componente esencial de la identidad coreana, la nación. La nación, no entendida como una mera entidad política, sino como comunidad imaginada. Tal como afirmó Benedict Anderson en su célebre ensayo Imagined Communities: «[...] For what we call nationalism operates on many levels and may be regarded as a form of culture as much as a species of political ideology and social movement» (Anderson 2006, 71). En efecto, es posible argumentar que todos estos elementos están presentes en el desarrollo del nacionalismo coreano. Si bien es posible encontrar las 
raíces del nacionalismo en elementos como el discurso filosófico de los académicos sirhak ${ }^{1}$ a finales de la dinastía Joseon (1392-1897) y, más adelante, en las corrientes de la ilustración y donghak ${ }^{2}$ que emergieron debido a factores tanto internos como externos (Shin 2000, 17), es posible afirmar que el nacionalismo coreano se desarrolla en gran medida como fuerte oposición a la agresión y dominación extranjera de la península que supuso el periodo colonial. Los intelectuales de la época utilizaron sus escritos para tratar de imbuir una consciencia nacional en el pueblo rescatando héroes históricos del pasado. En ocasiones el cine coreano se ha convertido en un amplificador de esas narrativas nacionalistas, como sucede con el drama histórico Myeongryang (2014), un ejemplo del cine épico que trata sobre las victorias navales del almirante Yi Sunsin (1545-1598) sobre los japoneses.

A finales de la década de los 90 la industria del cine en Corea del Sur experimentó una profunda transformación en un nuevo periodo que ha sido denominado "el renacimiento del cine coreano" tanto por los académicos como por los críticos. A través de este proceso de reconversión la industria logró competir con éxito en el mercado doméstico contra las producciones importadas de Hollywood, dando lugar a una acumulación de capital que facilitó el surgimiento de los "Korean blockbusters" o "taquillazos coreanos». El término puede definirse como un producto híbrido entre narrativas nacionales y lenguaje cinematográfico extranjero. Se considera que provee de algunos elementos «auténticos» que ayudan a la audiencia a creer y consumir las películas como "cine nacional" y "nuestro" (Kim 2016, 6). Este renacer del cine coreano estuvo caracterizado por un discurso de globalización a través de la localización. Después de la crisis del Fondo Monetario Internacional en 1998, hubo varias campañas en Corea del Sur que enfatizaron los sentimientos de unidad y comunidad de la cultura coreana. Por ejemplo, numerosas ONG participaron en la campaña de boicot a las producciones de Hollywood que surgió en parte como una respuesta a las presiones del Gobierno estadounidense para que los

1. Sirhak o «enseñanzas prácticas» fue una corriente filosófica de finales de la dinastía Joseon cuyos pensadores planteaban reformas para modernizar el país y cuya cosmovisión se alejaba del concepto de China como centro de la civilización promoviendo una visión de la historia más centrada en Corea. Sin embargo, el impacto del movimiento sería muy limitado debido a que la mayoría de sus pensadores se encontraban alienados del poder político.

2. Donghak o «enseñanzas del este» fue un movimiento fundado por Choe Je-u (18241864) que adquiriría dimensiones religiosas y políticas. En parte surgió como oposición a sohak o «enseñanzas de occidente», término con el que se conocía a las ideas que llegaban de Occidente, principalmente el catolicismo. 
coreanos redujesen el número de días de cuota en que las pantallas de cine se reservaban a la proyección de películas nacionales (Ok 2009, 39). La industria del cine acogió estos movimientos de forma activa canalizando su discurso nacionalista. De esta manera apeló a una parte de la identidad coreana relacionada con los orígenes. Parafraseando al poeta Rainer Maria Rilke «la verdadera patria del hombre es la infancia». Puede afirmarse que es ahí donde comienza un proceso de construcción de la identidad en el que no solo interviene lo intelectual o ideológico, sino también lo afectivo y lo cognitivo. En ese sentido, como se ha mencionado, el "Korean blockbuster" adopta técnicas de Hollywood y ofrece ámbitos y narrativas nacionales que remiten a la audiencia a códigos compartidos.

Sin embargo, es preciso añadir a esto que en el caso de las adaptaciones de obras extranjeras se adopta una estrategia que podríamos denominar de glocalización o "traducción cultural». Los productos resultantes apelan directamente a la nostalgia o los contextos comunes ofreciendo unos espacios abundantes en paisajes y aromas familiares que enfatizan la sensación de pertenencia a la comunidad e historia compartida.

Cabe destacar que la práctica de adaptar obras extranjeras a contextos locales no se originó en la industria del cine, sino que encuentra sus raíces en la literatura moderna de Corea (y más adelante se traslada al cine), en el género denominado pŏnan munhak (literatura de adaptación). Dos prominentes ejemplos de esta práctica son: Changhanmong de Cho Chunghwan, una adaptación coreana de 1913 del clásico Konjiki yasha de Ozaki Koyo, y Chinjutap de Kim Nae-sŏng, una novela serializada en la radio en 1947 y basada en El conde de Montecristo de Alejandro Dumas (Chung y Diffrient 2015, 46-47). Este tipo de obras han sido a menudo denostadas por los críticos, acuñando el término «copywood» en el caso del cine. Esto parte del prejuicio de considerar que una adaptación o remake es una mera copia o es inferior al original, cuando tiene el potencial de proveer respuestas intertextuales sobre importantes cuestiones como la nacionalidad, la ciudadanía o la participación colectiva en el cuerpo político en distintos momentos de la historia (ibid., 48). Además, es necesario señalar que si la adaptación se ha considerado a menudo bajo un prisma negativo esta tendencia aumenta cuando se trata de la adaptación al cine de obras literarias. Dentro de la comunicación entre ambas disciplinas el caso contrario (adaptación del cine a la literatura) es mucho más escaso, lo cual probablemente esté relacionado con una serie de prejuicios de los que deriva la visión de la adaptación como un producto inferior. Entre ellos quisiera destacar: la antigüedad de ambas disciplinas, siendo el cine muy reciente no ha gozado del mismo prestigio que la literatura, y lo que Robert Stam denomina parasitismo, la idea de que la relación del cine con la literatura 
es necesariamente parasitaria, cuando como afirmó Linda Hutcheon: «La adaptación es la manera en que las historias evolucionan y mutan para adaptarse a diferentes lugares y épocas” (Stam 2019, 66).

En relación con el concepto de glocalización y la forma en que la industria cinematográfica surcoreana adapta narrativas extranjeras a un contexto enteramente coreano en las siguientes secciones se ofrecerá un análisis de dos películas recientes, Burning (2018, dirigida por Lee Changdong) y The Handmaiden (2016, dirigida por Park Chan-wook), basadas en obras literarias de Haruki Murakami y Sarah Waters respectivamente.

\section{Tensión social EN El thriller De LeE Changdong: BuRning}

Burning (버닝, 2018) está basada en el relato "Barn Burning» ("Quemar graneros") publicado por Haruki Murakami en su colección de cuentos El elefante desaparece, quien a su vez tomó el título del relato homónimo de William Faulkner publicado en 1938. Si bien a priori ambos relatos pueden parecer muy diferentes desde el punto de vista del argumento, una lectura detenida puede revelar puntos de conexión entre ambos más allá del título y que nos remiten a cuestiones sobre la naturaleza humana. El relato de Murakami gira en torno a un hombre casado en la treintena que conoce a una chica más joven en la boda de un amigo y establece una relación de amistad con ella. Tras recibir dinero de una herencia, la chica decide viajar a Argelia y regresa con un novio japonés que ha conocido durante ese viaje. En uno de los encuentros con la pareja, el novio confiesa al protagonista mientras consumen marihuana que tiene costumbre de quemar un granero cada dos meses. Esto despierta la curiosidad del protagonista quien comienza a visitar los graneros de la zona tratando de averiguar cuál quemará la próxima vez. Durante un encuentro fortuito el nuevo novio le explica que ya ha quemado un nuevo granero a pesar de que el protagonista no ha sido capaz de descubrir cuál y también le informa de que hace tiempo que no ve a la chica. Al desplazarse a su casa en la última parte el protagonista encuentra otro nombre en el buzón y la chica parece haber desaparecido sin dejar rastro. El relato presenta un narrador homodiegético y focalización interna. Esta es una característica que trata de transmitirse de forma fiel en la adaptación en la que la historia se nos muestra desde la perspectiva del protagonista Jongu.

Sin embargo, la versión de Lee Changdong va más allá del material original en diversos aspectos. De hecho, una de las primeras cuestiones que sorprende al espectador es la manera en que el director crea un largometraje de más de dos horas a partir de un relato de tan solo algunas 
páginas. Dado que analizar adaptaciones supone priorizar la percepción para proveer algo más allá del comentario (Balwind Lind 2016, 22), me gustaría comenzar poniendo el foco sobre un factor estrechamente relacionado con el concepto de identidad, el uso del espacio. Si bien en las primeras líneas del relato de Murakami se aclara que la acción transcurre en Tokio, en su desarrollo las referencias al paisaje u elementos de la cultura japonesa son escasos. En cambio, el uso de los espacios en Burning parece cuidadosamente planeado por el director. Cuando se le preguntó en una entrevista por qué eligió Paju, a tan solo unos kilómetros de la frontera con Corea del Norte, como una de las localizaciones principales de la película, Lee respondió lo siguiente:

Paju has the kind of typical countryside in Korea that is disappearing more and more now. It's related to the past, and also related to the character of Jongsu's father [a farmer]. But it says something about a contemporary mindset too: the conflict between the South and the North is always in the subconscious for Koreans. (Lim 2018)

En efecto, el hanok o casa tradicional del padre del protagonista Jongu y los campos que la rodean nos remiten a un paisaje típico del ámbito rural coreano y tendrán un significado en la narración. Durante las primeras escenas mientras está haciendo recados el protagonista conoce a Haemi a la puerta de un comercio. Está trabajando junto a otra chica para promocionar el negocio bailando y ofreciendo a los transeúntes participar en un juego de lotería. Una escena familiar que podríamos encontrar en cualquier distrito comercial de Seúl. A continuación, fuman en un callejón durante el descanso de Haemi y es ella quien toma la iniciativa invitándolo a tomar algo. Se dirigen a un bar estilo pojangmacha ${ }^{3}$. Es en este contexto donde se desarrolla la escena en que Haemi muestra sus dotes de mímica pelando mandarinas que también aparece descrita en el relato de Murakami. La localización resulta simbólica porque la estética del bar nos remite a un icono del paisaje urbano coreano en peligro de extinción entre otras cosas por la falta de relevo generacional ${ }^{4}$. Descritas en el primer párrafo de la novela Seúl, invierno de 1964 de Kim Seungok, la aparición del establecimiento

3. Pojangmacha, literalmente "carro cubierto", son pequeñas tiendas que se montan en las calles en Corea y venden comida callejera, además de bebidas alcohólicas por las noches. Hoy día existen numerosas variantes que se instalan en locales por comodidad, pero utilizan el concepto pojangmacha en sus nombres como reclamo para indicar que ofrecen precios bajos y menús similares a los establecimientos originales.

4. Para más información sobre su desaparición progresiva véanse las entrevistas con distintos dueños de estos establecimientos en: PARK, Eddie: Pojangmacha. Tent of Nostalgia. 
tiene connotaciones relacionadas con la clase social. Si bien han evolucionado de manera que ya no sirven platos callejeros como el gorrión asado que menciona Kim Seungok en su obra y suelen situarse en locales, las cortinas de plástico que vemos detrás de Haemi durante toda la escena remiten a la audiencia a este contexto. Siguiendo el orden secuencial de la película, el siguiente escenario que se nos presenta es el oneroom ${ }^{5}$ de Haemi. El hecho de que su habitación esté situada colina arriba (barrios de renta baja en Seúl) tampoco es casual, sino que para el espectador coreano refuerza la identidad de clase de los personajes.

Esto se verá de forma más clara cuando entre en escena Ben, una suerte de Gatsby que conoce Haemi en su viaje a África, y se muestren los lugares que frecuenta en Seúl. Todas estas localizaciones aluden a identidades y forman un mosaico de "lo coreano" que la audiencia asocia a perfiles de clase determinados. Quizás es en ese punto en el que Lee establece una clara línea divergente en su reinterpretación de la obra de Murakami. Mientras que en el relato la relación entre el protagonista casado y la chica es descrita de forma ambigua como una amistad sin connotaciones sexuales, en la película el factor sexual es más explícito mostrando una escena de sexo entre Jongu y Haemi y otra en la que este se masturba en su habitación mientras ella está de viaje en África. De esta manera introduce una dinámica de relación muy diferente a la expuesta en el relato de Murakami a través del deseo de Jongu, quien además no está casado, sino que es un chico soltero de edad similar a Haemi. Lee Changdong afirmó lo siguiente en una entrevista sobre la película:

[...] what these projects had in common was the theme of anger -the anger and helplessness of young people today, and their sense that there is something wrong with the world that they don't quite realize or understand.

Es posible percibir cómo la ira toma un rol fundamental en la adaptación de Lee. De hecho, en la película hay un componente importante de tensión social apenas inexistente en la obra de Murakami. Esto se ve reflejado una vez más en el uso de los espacios narrativos. Las primeras escenas han reforzado la imagen de que tanto Jongu como Haemi son dos jóvenes en una situación económica precaria. Sin embargo, cuando entra en escena

En: Korea Exposé. https://www.koreaexpose.com/pojangmacha-tent-nostalgia/ [febrero, 2020].

5. Nombre que reciben en Corea los estudios de precios bajos que incluyen una cocina en la misma estancia y un pequeño baño. 
Ben se producirá un gran contraste. Jongu va a recogerlos en el aeropuerto en la vieja camioneta de su padre y van a un restaurante. Una vez salen de allí el coche de Ben (un deportivo de importación) está aparcado a la puerta del restaurante y tras intercambiar algunas palabras será Ben quien se encargue de llevar a Haemin a casa. Sin darse una discusión se hace ver al espectador que los personajes consideran este desenlace la opción más lógica (Lee 2018, 43:00). Por tanto, Jongu se ha visto desplazado por un nuevo novio adinerado. Esta diferencia de clase se marcará aún más en las siguientes escenas, ya que todos los lugares en los que aparecerá Ben nos remiten a paisajes de Seúl muy diferentes a los mostrados al principio de la obra y que denotan su alto poder adquisitivo. Esto se ve claro si, por ejemplo, comparamos el bar en que Jongu y Haemi comparten soju con el que aparece en el minuto 57:30 y en el que se muestra comida y vino occidental importado de un precio mucho mayor en Corea.

Una escena fundamental en el relato y la película ocurre cuando los tres personajes fuman marihuana juntos. También en la película Ben confiesa su extraña afición a Jongu, aunque los graneros han sido sustituidos por los invernaderos que hoy día pueblan los paisajes rurales de Corea. Sin embargo, en el caso de la película Haemi realiza una danza en topless frente a los dos hombres que representa «el gran hambre» y que en palabras del propio Lee constituye la escena más importante de la película (Lim 2018). Se trata de una referencia a la distinción que hacen los aborígenes del desierto de Kalahari entre "hambre pequeña" (la que se sacia con comida y bebida) y "gran hambre» (referida a la búsqueda de un significado en la vida) y que nos invita a la reflexión filosófica del significado de estos tres personajes.

Después Jongu la reprende por mostrar sus pechos frente a los hombres y esta será la última escena en que aparezca Haemi. En la novela el destino de la chica es incierto y misterioso dejando un final abierto. Sin embargo, en la película Jongu no solo trata de vigilar los invernaderos de la zona que podría quemar Ben, sino que al mismo tiempo intenta encontrar pistas que puedan llevarlo hasta Haemi. Al no dar con ella termina siguiendo los pasos de Ben y se introducen dos elementos muy significativos que dan pistas sobre el posible destino de Haemi. En primer lugar, cuando Jongu visita de nuevo el apartamento de Ben encuentra que el gato de la chica está viviendo allí. Sin embargo, el director va más allá y en otra escena en la que Jongu visita el baño encuentra una serie de objetos que parecen ser una colección de trofeos de diferentes mujeres con las que Ben habría mantenido relaciones, uno de los cuales pertenece a Haemi. Este elemento abre la posibilidad a la interpretación de que en realidad los invernaderos fuesen una metáfora de mujeres asesinadas a manos de Ben. Finalmente, el 
culmen de este triángulo amoroso con fuerte componente de clase llegará en la impactante escena final que nos remite de nuevo al título de la película, Burning. Un resultado de los sentimientos de ira, deseo y humillación de un joven aspirante a escritor frustrado de una familia empobrecida hacia un hombre de clase alta que puede permitirse todo tipo de extravagancias. De forma indirecta se sugiere que dichas extravagancias incluyen en su dimensión más macabra la posibilidad de abusar o disponer de las vidas de otras personas con menor poder adquisitivo de manera impune.

Si se toma en consideración la filmografía de Lee Changdong podría afirmarse que el conflicto social representado en Burning es hasta cierto punto característico, ya que busca con frecuencia en sus películas presentar la sociedad a través de las experiencias de individuos a menudo marginados o alienados y, por tanto, más vulnerables al abuso (Taylor Jones 2013, 76). Así, podría concluirse que el uso de espacios familiares en Burning no constituye únicamente un elemento relacionado con el concepto de consumer nationalism, sino que desde una perspectiva social sirve al doble propósito de realizar una crítica a la creciente desigualdad de la sociedad posmoderna surcoreana representada en personajes cuyos trasfondos arquetípicos forman también parte de "lo coreano".

\section{UNA ATMÓSFERA VICTORIANA EN LA COREA COLONIAL: LA DONCELLA}

Desde su irrupción en el panorama internacional con Oldboy (올드보이, 2003), Park Chan-wook ha sido considerado una figura polémica dentro de la industria del cine coreano, ya que sus trabajos a menudo contienen cierto grado de transgresión. En el caso de Oldboy, adaptó la narrativa de un manga japonés a la pantalla. Se trata de una película que combina técnicas cinemáticas deslumbrantes y culmina con una conclusión provocativa en la que juega con la moralidad convencional (o lo que se entiende por ella en la mayoría de las sociedades industrializadas) y abandona la dicotomía entre bueno y malo que caracteriza con frecuencia el thriller (Peirse y Martin 2013, 99).

La doncella (아가씨, 2016) no es una excepción a este carácter transgresor. Si bien esta adaptación de la novela Fingersmith de Sarah Waters es bastante fiel al hilo argumental de la obra (o, al menos, a buena parte), al igual que sucedía con Burning realiza una traducción cultural a un contexto puramente coreano. Ambientada en la década de los treinta, en el contexto de la Corea ocupada por los japoneses, la película narra una historia de amor entre dos mujeres, Lady Hideko (una noble japonesa que vive recluida por su tío en una mansión) y su nueva criada coreana, Sokhee, quien ha llegado a la mansión como parte de una conspiración secreta 
para convencer a Lady Hideko de que se case con un hombre y de esa manera puedan robar la fortuna que le corresponde por herencia. La mayor parte de la atención de la crítica hacia esta película se ha dirigido hacia las escenas altamente eróticas que representan la relación entre ambas mujeres. En un contexto coreano, conservador hacia la homosexualidad, llevar estas escenas a la gran pantalla suscitó polémicas. Consciente de ello, Park afirmó en una entrevista que:

Even if Korea as a whole won't agree to same-sex marriage, there is a point to keep on talking about it, and it is at a stage where it is enough so that when a film like this comes out, people finding it to be a shocking news. That's why I believe continuing to talk about the issue and making noise is important. (Kim 2016)

Cabe destacar que otras películas comerciales coreanas han tratado de forma sucinta relaciones homosexuales, si bien lo han hecho de un modo mucho más implícito que las representaciones que se han dado en el cine independiente. Ejemplos de ello podrían ser películas como The King and the clown (왕의 남자, 2005) o A girl at my door (도희야, 2014), en las que se presentan relaciones o personajes homosexuales, pero que no ofrecen escenas de sexo explícitas como las que aparecen en La doncella. En términos generales, las escenas de La doncella han sido recibidas de forma positiva por la audiencia, aunque también han suscitado críticas por parte de ciertos sectores que consideran que se llevaron a cabo para encajar con los estándares heterosexuales del sexo lésbico que retrata la industria pornográfica. (Armistead 2017)

En todo caso, siguiendo la línea de análisis de Burning quisiera poner el foco en la utilización de los espacios y el trasfondo. Posiblemente uno de los primeros elementos que llamará la atención a aquellos que conozcan Fingersmith es el cambio de la Inglaterra victoriana por la Corea colonial. No cabe duda de que el periodo colonial aún constituye uno de los más dolorosos de la historia reciente para los coreanos y al mismo tiempo un periodo oscuro, dado que muchos archivos japoneses de la preguerra aún permanecen cerrados (Cumings 2005, 139). Cuestiones como los crímenes de guerra y el colaboracionismo todavía obstaculizan las relaciones entre Corea y Japón. A pesar de ello, podría afirmarse que también es un periodo que suscita interés a juzgar por el gran número de películas ambientadas en la época que han estado apareciendo durante los últimos años. Algunos ejemplos serían: Assassination (암살, 2015), The last princess (덕혜옹 주, 2016), The Age of Shadows (밀정, 2016), The Battleship Island (군함도, 2017) o Anarchist from Colony (박열, 2017). 
Aunque las películas citadas pertenecen a distintos géneros (incluyendo el drama, biopic y película de acción), un común denominador es que todas ellas representan las dificultades y la lucha de los coreanos bajo la ocupación extranjera. Sin embargo, en el caso de La doncella podría afirmarse que este trasfondo histórico-político juega un papel secundario en la narrativa. Cuando se le preguntaron las razones por las que eligió este contexto particular para la película Park respondió:

"The original novel was set in the Victorian era," Park said. "When I thought about adapting it to the Korean Peninsula, I had no choice but to pick the Japanese colonial era. [...] It was the only period that encompassed a feudal order and strict class system [...] I needed a social hierarchy that allowed the existence of an heiress and handmaiden, but at the same time, the period had to be modern enough to have a mental clinic, which appears as a vital institution in the film.» (Jin 2016b)

De manera similar a lo que sucedía en Burning, las primeras escenas nos muestran a una Sok-hee de orígenes humildes con niños llorando ante su marcha que contrastan con la lujosa mansión de Lady Hideko en la que transcurre la mayor parte de la historia. La mansión es un escenario interesante en que Park recrea con éxito un estilo victoriano en el contexto de la Corea colonial, se trata de una mezcla entre estilos de arquitectura occidentales y orientales que combina ostentosos salones amueblados al estilo europeo con habitaciones con tatami y puertas correderas. Los muebles y vestimentas resultan claves para reforzar la ambientación. Otras localizaciones incluyen elementos relacionados con la modernidad como coches, trenes o edificios de estilo occidental. De entre las estancias de la mansión destaca la biblioteca como un lugar central en el argumento tanto de la película como de la novela. Al igual que sucedía en la biblioteca del tío de Maud en Fingersmith, la de la película contiene una colección pornográfica de Kouzuki, quien obliga a Lady Hideko a leer para otros hombres en reuniones. Esta biblioteca es además un símbolo de una gran riqueza alcanzada a través de la colaboración con los japoneses simbolizando de este modo la opresión personal y también política (Park et al. 2019, 185).

Si bien en palabras del director la localización y el marco histórico de la película obedecen a cuestiones pragmáticas, como ya vemos es una decisión que tiene un impacto trascendental en determinados elementos de la trama. Por ejemplo, la relación entre Lady Hideko y Sook-hee ya no es solo la relación entre dos mujeres de distinta clase social, sino que el hecho de que una sea japonesa y la otra coreana en el contexto colonial implica otras connotaciones en las dinámicas de poder, lo cual se ve reflejado en que las criadas deban adoptar nombres japoneses y utilizar dicho idioma. Igual de interesante es la figura del tío Kouzuki, quien ni es japonés (a 
pesar de haber adoptado un nombre japonés) ni de la nobleza. Representa el tipo de personaje egoísta que dejando de lado cuestiones morales busca aprovecharse de la situación de Corea para su beneficio personal. En una escena significativa en la que Kouzuki mantiene una conversación con el conde, este último pregunta «¿Por qué esa necesidad de convertirte en japonés?», a lo que responde: "Porque Corea es fea y Japón es bonita». Cuando se preguntó a Park por esta escena respondió lo siguiente:

There are those people in Korean society, found among the intellectuals in the upper class, who during the Japanese occupation would worship the Japanese. These days, they could be worshiping the Americans, and some of them might be worshiping the French or the Germans. And above all else, prior to the Japanese occupation, the Koreans have been worshiping the Chinese for hundreds of years, and that's something that had become part of the conscience of some section of the ruling class and some of the intellectuals of the day. There's a Korean term, sadaejuui, that is used to uniquely express this notion, where the people of a smaller nation are so drawn to the power of a larger nation, and become subservient to that power. They internalize it so much that they are not worshiping the bigger power by force, but are doing it voluntarily. Through the character of Uncle Kouzuki, I wanted to paint a portrait of these poor, sad, and pathetic individuals-who are poor, I say-but who become a big threat and a serious danger for the other people of their nation. (Topalovic 2016)

Este tipo de personaje, el colaborador, apela de una forma muy particular a la memoria colectiva de los coreanos sobre este periodo. Esto es porque, tal como afirma Park, es un tipo de personaje que ha sido una constante a lo largo de la historia de Corea. Tras finalizar la Segunda Guerra Mundial el asunto del colaboracionismo estuvo envuelto en un manto de silencio y secretismo durante décadas. La razón principal para ello es que jugaba en contra de los intereses de una élite colonial que mantendría su poder en alianza con Syngman Rhee (De Ceuster 2012, 208). Por ello, durante el periodo de democratización hubo una reevaluación de las actividades colaboracionistas, que ya eran de sobra conocidas por los coreanos. La dimensión colaboracionista de Kouzuki encuentra su resonancia en la literatura coreana, autores como Chae Mansik ya criticaban el egoísmo de los que buscaban solo su enriquecimiento material en el contexto colonial en obras célebres como Paz bajo el cielo (태평천하, 1938) o Mi tío el idiota (치숙, 1938). Más tarde, una vez finalizada la guerra, seguirían representándose personajes que pasaron de servir a los japoneses a los americanos, como por ejemplo el doctor Lee en Kapitan Lee (꺼삐딴 리, 1962) de Chon Kwangyong. Por tanto, es posible afirmar que este tipo de personajes también forman parte del paisaje presente en el imaginario colectivo 
de los coreanos de generaciones posteriores que no vivieron el periodo colonial, pero aprendieron sobre el mismo a través del testimonio de sus mayores, la historia y las múltiples representaciones artísticas de la época. Es importante enfatizar que La doncella no retrata las relaciones de poder únicamente en términos de clase, nacionalidad o idioma, sino también de género. Ambas protagonistas son mujeres manipuladas por sus homólogos masculinos para sus fines, sin embargo, la escena final en la que disfrutan de su amor en libertad tiene un significado de empoderamiento. De hecho, en una entrevista Park citó la escena en la que ambas saltan el muro de la mansión dándose cuenta por primera vez de que realmente no era tan alto como el momento más importante de la película en términos de mensaje (Topalovic 2016). Porque es el momento simbólico en que las dos caen en la cuenta de que las barreras a su libertad eran de una naturaleza más psicológica que física. En ese sentido, un momento de liberación crucial para Hideko ocurre cuando destruye por completo la colección pornográfica de Kouzuki que era obligada a leer en frente de hombres de la alta sociedad.

Como se ha mencionado, otro elemento interesante es el idioma. Numerosas películas ambientadas en el periodo colonial recurren al uso de coreano y japonés incluyendo subtítulos en hangul para este último. Por ello es frecuente que la audiencia coreana esté acostumbrada a escuchar japonés en este tipo de películas. Uno de los motivos por los que suele incluirse es para dotarlas de un mayor realismo e inmersión. Sin embargo, en este caso también resulta significativa la manera en que todas las conversaciones entre Hideko y Sokhee ocurren en coreano y cómo esto contrasta con el uso del japonés cuando están en presencia de los personajes masculinos. De esta manera el lenguaje sirve como herramienta para enfatizar la distancia entre los poderosos y los manipulados adquiriendo una nueva dimensión. En relación con esto la autora de Fingersmith, Sarah Waters, comentó lo siguiente en una entrevista:

The novel is about class rather than gender: people passing themselves off as something they're not. The film is more about colonialism: that very fraught relationship between Korea and Japan. (Armitstead 2017)

\section{COnClusiones}

A pesar de las muchas diferencias que presentan las dos adaptaciones analizadas podría categorizarse a ambas como thrillers psicológicos con elementos comunes en lo referente al proceso de adaptación que realizan. Una de las cuestiones que llama la atención al comparar ambas películas 
es la forma en que realizan traducciones culturales de los contextos originales a espacios totalmente coreanos que apelan a códigos conocidos por la audiencia coreana.

Por supuesto, como se ha aclarado en la introducción, no se trata de un fenómeno nuevo, sino que desde el resurgir del cine coreano a finales de los 90 se ha venido utilizando un sistema híbrido que imita el lenguaje cinematográfico de Hollywood ofreciendo narrativas locales. A esto añadimos en el caso de las adaptaciones la manera en que historias extranjeras son trasladadas a contextos coreanos. Esto responde a varias razones, más allá de cuestiones pragmáticas también está relacionado con el potencial de apelar a códigos compartidos por la audiencia doméstica para asegurar su éxito y también con las dinámicas de adaptación presentes en el cine y la literatura coreanos desde hace décadas.

En ambos casos se ha enfatizado el uso de los espacios que hacen ambos directores para llevar a cabo esta estrategia de glocalización, aunque como se ha podido observar esta no es la única manera en la que se apela a la identidad cultural. A través de la adaptación de literatura extranjera ambas películas introducen elementos que están relacionados con problemas contemporáneos de la sociedad surcoreana. En el caso de Burning, un joven de orígenes humildes incapaz de encontrar su lugar en una sociedad altamente competitiva y que contrasta con el estilo de vida de Ben representa la creciente desigualdad en la sociedad, mientras que en el caso de La doncella, aparte de su descripción transgresora y gráfica del romance homosexual entre dos mujeres para una sociedad aún conservadora en esta materia, nos remite también de forma más indirecta a problemas aún sin resolver del legado colonial como lo es el colaboracionismo. De esta manera es posible argumentar que ambas adaptaciones dan lugar a nuevos productos que se integran y juegan un papel en lo que entendemos como K-cinema, una de las dimensiones de la llamada ola coreana o ballyu. Sin embargo, el propio concepto continúa siendo controvertido para muchos académicos. Por ejemplo, se ha argumentado que la hibridación del cine coreano ha fallado en crear un tercer espacio cultural porque las películas han abandonado el espacio sociocultural coreano para ajustarse a los gustos occidentales (Jin 2016a, 89). En relación con esto, otros autores como Kim Kyung Hyun han criticado la falta de variedad de las producciones cinematográficas recientes en Corea (Kim 2011). Quizás esto también esté relacionado con los prejuicios existentes en torno a las adaptaciones que se han descrito en la introducción de este trabajo, aunque como se ha podido comprobar en este caso ambas películas van más allá de los materiales de origen creando obras totalmente nuevas que no solo se trasladan a un 
contexto coreano, sino que cuentan con nuevas dimensiones que no estaban presentes en las obras literarias que adaptan.

En un contexto de creciente globalización el concepto hegemónico de nacionalismo étnico coreano está siendo desafiado por los flujos migratorios y al mismo tiempo la industria del cine está sufriendo numerosas transformaciones relacionadas con su transnacionalización, por lo que la manera en que se adaptará y evolucionará resulta incierta. Sin embargo, independientemente de ello, parece claro que el cine continuará desarrollándose junto con la identidad coreana se trate o no de adaptaciones. Ambos elementos parecen constituir un tándem indisoluble y la identidad no puede ser comprendida sin una serie de elementos culturales internalizados que «nos» distinguen del «otro» (Giménez 2012, 1).

\section{BiBliografíA}

ANDERSON, Benedict. Imagined Communities. Reflections on the Origin and Spread of Nationalism. Verso, 2006.

ARMISTEAD, Claire. "Sarah Waters. The Handmaiden turns pornography into a spectacle but its true to my novel'». The Guardian, 2017. https://www.theguardian. $\mathrm{com} /$ film/2017/apr/08/sarah-waters-the-handmaiden-turns-pornography-intoa-spectacle-but-its-true-to-my-novel- [febrero 2021].

BALDwind Lind, Paula (ed.). Telling and Re-telling Stories. Studies on Literary Adaptation to Film. Cambridge Scholars Publishing, 2016.

Chung, Hye Seung y David ScotT Diffrient (eds.). Movie Migrations. Transnational Genre Flows and South Korean Cinema. Rutgers University Press, 2015.

Cumings, Bruce. Korea's Place in The Sun. A Modern History. W. W. Norton \& Company Inc., 2005.

De Ceuster, Koen. "The Nation Exorcised. The Historiography of Collaboration in South Korea». Korean Studies, 2002, volume 25, n. ${ }^{\circ} 2$.

GiméNEZ, Gilberto. La cultura como identidad y la identidad como cultura. Instituto de Investigaciones Sociales de la UNAM, 2012.

Jin, Dal Yong. 2016a New Korean Wave. University of Illinois Press, 2016.

Jin, Eun Soo. 2016b. "The Handmaiden generates a buzz". Korea Joongang Daily, 03.05.2016. http://koreajoongangdaily.joins.com/news/article/article. aspx?aid=3018243 [febrero 2021].

KIM, Kristen Yonsoo. "The Handmaiden's Explicit Lesbian Sex is shaking up Korean Cinema». 19.10.2016. https://www.complex.com/pop-culture/2016/10/ park-chan-wook-the-handmaiden-lesbian-sex-scenes [febrero 2021].

KIM, Kyung Hyun. Virtual Hallyu. Korean Cinema of the Global Era. Duke University Press, 2011. 
KIM, Sun Kyung: "Renaissance of Korean National Cinema as a Terrain of Negotiation and Contention between the Global and the Local: Analysing two Korean Blockbusters, Shiri (1999) and JSA (2000)». Universidad de Essex, 2006.

Lim, Dennis. Interview: Lee Changdong. En: Film Comment. Film Society of Lincoln Center. 2018. https://www.filmcomment.com/blog/cannes-interview-lee -chang-dong/

OK, Hyeryoung. "The Politics of the Korean Blockbuster. Narrating the Nation and the Spectacle of 'Glocalisation' in 2009 Lost Memories". Transnationalism and Film Genres in East Asian Cinema. Spectator, Fall 2009, 29:2, pp. 37-47.

PARK, Eddie: Pojangmacha. Tent of Nostalgia. En: Korea Exposé. https://www. koreaexpose.com/pojangmacha-tent-nostalgia/ [febrero, 2020].

PARK, Heebon, Julie SANDERS y Moonyoung CHUNG. "Secondary Pleasures, Spatial occupations and Postcolonial Departures. Park Chan-wook's Agassi/The Handmaiden and Sarah Waters's Fingersmith». Neo-Victorian Studies, 2019, 11:2, pp. 177-205.

PeIRSE, Alison y Daniel MARTin (eds.). Korean Horror Cinema. Edinburgh University Press, 2013.

SHIN, Yong-ha. Modern Korean History and Nationalism. Traducido al inglés por NM. Pankaj. Jimoondang, 2000.

STAM, Robert. World Literature, Transnational Cinema and Global Media. Towards a Transartistic Commons. Routledge, 2019.

TAYlor Jones, Kate E. Rising Sun, Divided Land. Japanese and South Korean Filmmakers. Wallflower Press, 2013.

Topalovic, Goran. «Interview: Park Chan-wook». Filmcomment, 28.10.2016. https:// www.filmcomment.com/blog/interview-park-chan-wook/ [febrero 2021].

\section{FILMOGRAFÍA}

LEE, Changdong. Burning. CGV Arthouse, 2018.

PARK, Chan-wook. The Handmaiden. CJ Entertainment, 2016. 